Letter to the Editor

\title{
Ultrasound and screening tool for dengue fever
}

\author{
Beuy Joob ${ }^{1}$, Viroj Wiwanitkit ${ }^{2}$ \\ ${ }^{1}$ Medical Academic Centre, Bangkok, Thailand \\ ${ }^{2}$ DY Patil University, Pune, India
}

\section{Dear Editor,}

We read the publication "Ultrasound is not useful as a screening tool for dengue fever" with great interest. Parmar et al. noted that "increased gall bladder wall thickness, pleural effusion, ascites, hepatomegaly, and splenomegaly are highly suggestive of dengue fever in clinically suspected cases. However, ultrasound should not be used as a screening tool, as negative ultrasound findings cannot rule out dengue fever due to the low sensitivity of this examination [1]". We would like to share our ideas and experience on using ultrasound for diagnosis of dengue in our setting, tropical Indochina, where dengue is highly prevalent. Ultrasound is not routinely recommended for diagnosis of dengue. The basic screening tool is the tourniquet test, which is helpful for diagnosis of thrombocytopaenia [2]. The abdominal ultrasound investigation in dengue patients usually gives a negative finding. Positive findings are usually observed in severe cases in which a simple clinical examination combining simple laboratory investigations, complete blood count, and dengue immunological test can help in the diagnosis. Also, the mentioned positive findings, such as thickening gall bladder or hepatomegaly, can also be seen in other tropical infections such as viral hepatitis [3]. It can be concluded that ultrasound is not appropriate and not cost effective for screening of dengue fever.

\section{Conflict of interest}

The authors report no conflict of interest.

\section{References}

1. Parmar J, Mohan C, Kumar GP, Vora M. Ultrasound is not useful as a screening tool for Dengue fever. Pol J Radiol 2017; 82: 693-700.

2. Wiwanitkit $\mathrm{V}$. The tourniquet test is still a good screening tool for dengue illness. Trop Doct 2005; 35: 127-128.
3. Joob B, Wiwanitkit V. Letter to Editor Commenting on "Patterns of Gall Bladder Wall Thickening in Dengue Fever". Ultrasound Int Open 2017; 3: E165. 